\title{
Perfil e fatores causais de prematuridade em recém-nascidos atendidos em um centro de reabilitação infantil no interior de Minas Gerais
}

\author{
DOI: 10.47224/rm.v5i10.84
}

\author{
Gisélia Gonçalves de Castro \\ Bruna da Silva Araújo \\ lêda Pereira Magalhães Martins \\ Juliana Gonçalves Silva de Mattos
}

e-mail: giseliagcastro@gmail.com

\begin{abstract}
Resumo
Caracterizou-se o perfil dos recém-nascidos prematuros que estiveram em reabilitação no setor de Fisioterapia Pediátrica no interior de Minas Gerais. Estudo analítico, descritivo e retrospectivo, realizado por análise de prontuários de 20152018. A análise dos dados foi descritiva. Analisou-se 63 prontuários sendo a maioria de meninos (52,4\%). Os partos aconteceram com tempo gestacional $\geq 29$ semanas $(77,8 \%)$, com $\mathrm{RN}$ 's de baixo peso $(44,4 \%)$, com bons resultados de Apgar no primeiro e quinto minuto e com perímetro cefálico entre 28 e $33 \mathrm{~cm}$ (42,8\%). Grande parte dos partos foram cesariados $(63,5 \%)$, com prematuros moderados $(44,4 \%)$, com maior incidência de meninas por parto vaginal $(12,7 \%)$ e meninos por parto cesariado (36,5\%). As causas da prematuridade mais evidentes foram por ruptura da bolsa e Préeclâmpsia/Eclâmpsia/Síndrome de Helps (17,5\%, respectivamente). Encontrou-se alterações no teste do olhinho (39,7\%). O tempo médio do encaminhamento médico até a primeira avaliação fisioterapêutica foi de 57,4 dias, enquanto até a alta fisioterapêutica foi de 339,8 dias, sendo a desistência o grande motivo (50,8\%). Percebeu-se incompletude dos dados nos prontuários, sendo necessários trabalhos pontuais de educação continuada e orientações quanto a importância do preenchimento, além de orientação às famílias quanto a adesão e acompanhamento ao tratamento de reabilitação.
\end{abstract}

Palavras-chave: Saúde Infantil; Nascimento Prematuro; Fisioterapia.

\begin{abstract}
The profile of premature newborns who were undergoing rehabilitation in the Pediatric Physiotherapy sector in the interior of Minas Gerais was characterized. Analytical, descriptive and retrospective study performed by analyzing medical records from 2015-2018. Data analysis was descriptive. 63 medical records were analyzed, most of them boys (52.4\%). The deliveries took place with gestational time $\geq 29$ weeks (77.8\%), with low birth weight newborns (44.4\%), with good Apgar results in the first and fifth minutes and with head circumference between 28 and $33 \mathrm{~cm}(42,8 \%)$. Most births were cesarean (63.5\%), with moderate premature babies (44.4\%), with a higher incidence of girls due to vaginal delivery (12.7\%) and boys due to cesarean delivery (36.5\%). The most evident causes of prematurity were rupture of the sac and Preeclampsia / Eclampsia / Helps Syndrome (17.5\%, respectively). Alterations were found in the eye test (39.7\%). The average time from medical referral to the first physical therapy assessment was 57.4 days, while until physiotherapy discharge it was 339.8 days, with dropout being the main reason (50.8\%). Incomplete data was perceived in the medical records, requiring specific continuing education work and guidance on the importance of filling it out, as well as guidance to families on adherence and monitoring of rehabilitation treatment.
\end{abstract}

Keywords: $\quad$ Child Health, Premature Birth, Physiotherapy.

\section{INTRODUÇÃO}

A prematuridade pode ser definida como gestação interrompida antes de 37 semanas de gestação, por vários motivos. Pode ser classificada como prematuridade limítrofe (com nascimento do recémnascido entre 35 e 36 semanas de gestação), moderada (entre 31 e 34 semanas) e extrema (com nascidos antes das 30 semanas de gestação). O recém-nascido (RN) ainda pode ser identificado como 
pré-termo (nascidos antes de 37 semanas de gestação), a termo (nascidos entre 37 e 42 semanas de gestação) e pós- termo (nascidos com mais de 42 semanas de gestação) (SCOPEL, 2017).

A taxa de prematuridade é analisada pela quantidade de nascidos vivos prematuros divididos pelo número total de nascimentos, sem esses nascidos vivos ou natimortos (MENETRIER; ALMEIDA, 2016).

Os RN's prematuros normalmente apresentam regresso no desenvolvimento neuropsicomotor e alterações no SNC (sistema nervoso central), devido à vulnerabilidade dos mesmos, diminuindo a sobrevida desses neonatos e aumentando a preocupação com as possíveis consequências (FERNANDES et al., 2017).

Cita-se como consequências as lesões neurológicas, sendo a paralisia cerebral (PC) a mais recorrente, que resultam em alterações cognitivas, motoras e de aprendizagem. Em decorrência dessas lesões há a exposição dos RN's prematuros a vários fatores de risco como a idade gestacional, as doenças maternas ou uso de drogas ilícitas pela mãe, o tabagismo, a nutrição inadequada, as deformações placentárias, o histórico de partos prematuros pregressos, o baixo Apgar nos primeiros minutos de vida, as infecções, as hemorragias cerebrais e a hipóxia neonatal (BONFIM et al., 2016).

Diante do exposto, é necessário a identificação dessas variáveis de forma precoce para que inclua na vida desses RN's prematuros um tratamento para o desenvolvimento neuropsicomotor prevenindo ou diminuindo as sequelas (BONFIM et al., 2016).

Autores reconheceram que melhores resultados de intervenção são obtidos quando há estimulação fisioterapêutica precocemente a fim de reduzir as alterações já encontradas e os padrões patológicos já instalados (MEDEIROS et al., 2009).

Sabendo que o atendimento fisioterapêutico precoce aos RN's prematuros garante uma melhor qualidade de vida (QV), evitando/reduzindo possíveis sequelas advindas da idade gestacional interrompida, esse estudo objetiva analisar o perfil dos RN's prematuros, em relação às causas do parto prematuro, tipo de parto, condições do RN e tempo de encaminhamento ao Centro de Reabilitação Infantil.

\section{METODOLOGIA}

Trata-se de um estudo descritivo e retrospectivo referente ao período de 2015 a 2018 em um ambulatório no município em Minas Gerais (MG).
Foram coletados dados através da análise de prontuários dos recém-nascidos prematuros que estiveram em reabilitação no setor de Fisioterapia Pediátrica do Centro de Saúde no referente período. Coletou-se as variáveis idade atual, sexo, idade gestacional (IG), peso, tipo de parto, valores de Apgar, medida do perímetro cefálico, complicações ao nascimento, bem como as datas de encaminhamentos médico, avaliação e alta fisioterapêutica.

A análise descritiva dos dados foi por meio de frequências absolutas $(N)$ e relativas (\%) para os dados categóricos e média e desvio padrão para os dados numéricos. Os dados foram compilados e tabulados no Excel e analisados pelo Software Statiscal Package for Social Sciences (SPSS) versão 18.0 para Windows.

O presente trabalho se encontra de acordo com a Resolução 466/12 do Conselho Nacional de Saúde, sendo submetido à avaliação do Comitê de Ética em Pesquisa do UNICERP (COEP/UNICERP) com aprovação número 20181450 FIS010.

\section{RESULTADOS E DISCUSSÃO}

Foram coletados dados de 63 recém-nascidos prematuros que estavam em reabilitação no período entre 2015 a 2018, sendo 52,4\% meninos e 47,6\% meninas.

Os partos ocorreram em sua maioria com tempo gestacional superior a 29 semanas $(77,8 \%$, Tabela 1$)$, corroborando com outros estudos (CASTRO; FIGUEIREDO; CANO, 2016). Ainda, a maioria nasceu com baixo peso $(44,4 \%)$, sendo o sexo masculino mais evidente $(23,8 \%)$, discordando dos principais achados na literatura (NASCIMENTO; GOTLIEB, 2001; VIANA et al., 2013).

Em relação ao Apgar no primeiro e quinto minuto de vida a maioria dos RN's apresentaram bons resultados $(71,4 \%$ no primeiro minuto; $81,0 \%$ no quinto minuto). Teve-se melhoras significativas naqueles que apresentaram asfixia moderado/grave evoluindo de $11,1 \%$ no primeiro minuto de vida para $1,6 \%$ no quinto minuto, sendo semelhantes ao estudo de Nunes; Bertuol; Siqueira (2016). Ainda, o perímetro cefálico (PC) mais evidente foi entre 28 e $33 \mathrm{~cm}(42,8 \%)$. 
Tabela 1 - Caracterização dos RN's prematuros quanto a Idade Gestacional ao nascer (em semanas), ao peso, Apgar no 10 e no 5o minuto e Perímetro Cefálico. 2015 a 2018 em um ambulatório no município em Minas Gerais (MG).

\begin{tabular}{|c|c|c|c|}
\hline \multicolumn{3}{|c|}{ Variável } & \multirow{2}{*}{$\begin{array}{c}\text { Total }(n=63) \\
\text { FA (\%) } \\
10(15,9 \%)\end{array}$} \\
\hline \multirow{3}{*}{$\begin{array}{l}\text { Idade Gestacional } \\
\text { nascer (em semanas) }\end{array}$} & \multirow{3}{*}{ ao } & 15 a 28 semanas & \\
\hline & & $\geq 29$ semanas & $49(77,8 \%)$ \\
\hline & & Não informado & $04(6,3 \%)$ \\
\hline \multirow{6}{*}{ Peso* } & & Normal & $01(1,6 \%)$ \\
\hline & & Insuficiente & $09(14,3 \%)$ \\
\hline & & Baixo peso ao nascer & $28(44,4 \%)$ \\
\hline & & Muito baixo peso ao nascer & $14(22,2 \%)$ \\
\hline & & Extremo baixo peso & $06(9,5 \%)$ \\
\hline & & Não informado & $05(8,0 \%)$ \\
\hline \multirow{4}{*}{ Apgar 19 minuto } & & Asfixia grave & $03(4,8 \%)$ \\
\hline & & Asfixia moderada & $04(6,3 \%)$ \\
\hline & & Bom estado & $45(71,4 \%)$ \\
\hline & & Não informado & $11(17,5 \%)$ \\
\hline \multirow{4}{*}{ Apgar 50 minuto } & & Asfixia grave & $00(-)$ \\
\hline & & Asfixia moderada & $01(1,6 \%)$ \\
\hline & & Bom estado & $51(81,0 \%)$ \\
\hline & & Não informado & $11(17,4 \%)$ \\
\hline \multirow{4}{*}{ Perímetro cefálico } & & 22 a $27 \mathrm{~cm}$ & $13(, 0 \%) 20,6 \%$ \\
\hline & & 28 a $33 \mathrm{~cm}$ & $27(42,8 \%) 42,9 \%$ \\
\hline & & $\geq 34 \mathrm{~cm}$ & $5(7,9 \%)$ \\
\hline & & Não informado & $18(28,6 \%)$ \\
\hline
\end{tabular}

Tabela 2 - Distribuição dos RN's prematuros quanto ao tipo de parto, quanto à classificação e causa da prematuridade. 2015 a 2018 em um ambulatório no município em Minas Gerais (MG).

\begin{tabular}{|c|c|c|}
\hline & Variável & $\begin{array}{l}\text { TOTAL } \\
(n=63) \\
\text { FA (\%) }\end{array}$ \\
\hline \multirow{3}{*}{ Tipo de Parto } & Vaginal & $14(22,2 \%)$ \\
\hline & Cesáreo & $40(63,5 \%)$ \\
\hline & Não informado & $09(14,3 \%)$ \\
\hline \multirow{4}{*}{$\begin{array}{l}\text { Classificação de } \\
\text { Prematuridade** }\end{array}$} & Limítrofe & $14(22,2 \%)$ \\
\hline & Moderado & $28(44,4 \%)$ \\
\hline & Extremo & $17(27,0 \%)$ \\
\hline & Não informado & $04(6,4 \%)$ \\
\hline \multirow{9}{*}{ Maturidade*** } & Ruptura de bolsa & $11(17,5 \%)$ \\
\hline & Causas desconhecidas & $02(3,2 \%)$ \\
\hline & Gemelaridade & $07(11,1 \%)$ \\
\hline & Sofrimento fetal & $03(4,8 \%)$ \\
\hline & Ausência/perda de líquido amniótico & $03(4,8 \%)$ \\
\hline & $\begin{array}{l}\text { Pré-eclâmpsia/Eclâmpsia/Síndrome de } \\
\text { Helps }\end{array}$ & $11(17,5 \%)$ \\
\hline & Infecções do Trato Urinário & $02(3,2 \%)$ \\
\hline & Trabalho de parto prematuro & $03(4,8 \%)$ \\
\hline & Não informado & $22(36,5 \%)$ \\
\hline
\end{tabular}

***Não são mutuamente simultâneos, podendo haver mais de uma causa para a prematuridade. 
Grande parte dos partos foram cesariados $(63,5 \%$, Tabela 2) equiparando com o panorama nacional e contrariando a indicação da Organização Mundial de Saúde (OMS) que preconiza que um total de $15 \%$ dos partos sejam de cesárea. O Brasil é o segundo país que mais realiza partos cesarianos no mundo com um índice de 55,6\% (HOLANDA et al., 2015; PINTO et al., 2019).

Em relação ao grau de prematuridade, a maioria foi classificada como prematuros moderados $(44,4 \%)$, com maior incidência de meninas por parto vaginal $(12,7 \%)$ e meninos por parto cesariado $(36,5 \%)$. Podese observar que $14,3 \%$ dos prontuários não continham a informação do tipo de parto, evidenciando a incompletude da Caderneta de Saúde da Criança (CSC).

As causas da prematuridade mais evidentes foram a ruptura da bolsa e a Préeclâmpsia/Eclâmpsia/Síndrome de Helps (17,5\%, respectivamente) e a gemelaridade $(11,1 \%)$. Outros autores identificaram as mesmas causas (ABRAHÃO et al., 2015; OLIVEIRA; MATTOS, 2016; CASTRO et al., 2019). A pré-eclâmpsia é uma das adversidades mais comuns durante $\mathrm{o}$ período gestacional entre brasileiras, representando 10,0\% (OLIVEIRA; VIEIRA, 2015).

Foram analisados os resultados dos testes de triagem como o do pezinho, da orelhinha e do olhinho. Esses testes são preconizados e disponibilizados pelo Ministério da Saúde (MS) desde 2001, 2010 e 2007, respectivamente (MENEZES et al., 2016; MS, 2015) e estão inclusos no Programa Nacional de Triagem Neonatal (PNTN) desde 2001 com a intenção de detectar, precocemente, patologias e disfunções no recém- nascido e dessa forma, identificar o tratamento ideal para os bebês garantindo assim, uma melhor qualidade de vida a eles (MS, 2016). Apesar do teste da orelhinha ser imposto pelo MS ainda há RN's que não o realizam, como mostra a Tabela 3.

Tabela 3 - Resultados da triagem neonatal realizada nos RNS prematuros. 2015 a 2018 em um ambulatório no município em Minas Gerais (MG).

\begin{tabular}{|c|c|c|c|}
\hline \multicolumn{2}{|c|}{ Variável } & \multirow{2}{*}{$\frac{\text { Masculino }(n=33)}{18(28,6 \%)}$} & \multirow{2}{*}{$\frac{\text { Feminino }(n=30)}{14(22,2 \%)}$} \\
\hline Tocto do nozinho & Normal & & \\
\hline 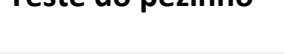 & Não informado & $15(23,8 \%)$ & $16(25,4 \%)$ \\
\hline \multirow{3}{*}{ Teste da orelhinha } & Normal & $17(27,0 \%)$ & $18(28,6 \%)$ \\
\hline & Não realizado & $01(1,6 \%)$ & $00(-)$ \\
\hline & Não informado & $15(23,8 \%)$ & $12(19,0 \%)$ \\
\hline \multirow{3}{*}{ Teste do olhinho } & Normal & $13(20,6 \%)$ & $12(19,0 \%)$ \\
\hline & Alterado & $04(6,3 \%)(6,4 \%)$ & $01(1,6 \%)$ \\
\hline & Não informado & $16(25,4 \%)$ & $17(27,0 \%)$ \\
\hline
\end{tabular}

Fonte: os autores

O teste com menor percentual de normalidade nos resultados foi o teste do olhinho $(39,7 \%)$, revelando alterações como retina imatura $(3,2 \%)$, estrabismo e deslocamento de retina (1,6\%, respectivamente).

Contudo, nota-se uma grande quantidade de dados incompletos presentes nos prontuários, que são advindos da CSC, de onde foram coletadas as informações. Estudos mostram a falha de profissionais de saúde em relação à completude dos dados da criança e ainda, que o teste do pezinho e do reflexo vermelho (ou teste do olhinho) está entre os dados menos preenchidos na caderneta, com 14,1\% e 14,2\%, respectivamente (ABUD; GAIVA, 2016).

A CSC é um documento que deve acompanhar o RN desde as primeiras consultas até os 10 anos de idade, por conter informações sobre a gravidez, o parto e as condições que o bebê se encontrou ao nascer (GAÍVA; SILVA, 2014). Quando preenchido corretamente auxilia os profissionais de saúde quanto às devidas precauções, melhor conduta terapêutica, continuidade de tratamento/acompanhamento, bem como conhecimento do histórico médico do RN.

Diante do cenário exposto, verificou-se o tempo gasto da data do encaminhamento médico até a data da avaliação fisioterapêutica, da data de avaliação fisioterapêutica até a data da alta e da data do encaminhamento médico até a data da alta fisioterapêutica ( Tabela 4) 
Tabela 4 - Cálculo dos períodos, em meses, entre a data de encaminhamento, a data de avaliação e a data de alta dos RN's prematuros. 2015 a 2018 em um ambulatório no município em Minas Gerais (MG).

\begin{tabular}{|c|c|c|c|}
\hline Período em dias & $\begin{array}{l}\text { Data do encaminhamento até a } \\
\text { data da avaliação* }\end{array}$ & $\begin{array}{l}\text { Data da avaliação } \\
\text { até a data de alta } \\
\text { ou interrupção } \\
\end{array}$ & $\begin{array}{l}\text { Data do encaminhamento } \\
\text { até a data da alta ou } \\
\text { interrupção* }\end{array}$ \\
\hline & FA (\%) & FA (\%) & FA (\%) \\
\hline Até um mês & $18(28,6 \%)$ & $09(14,3 \%)$ & $02(3,2 \%)$ \\
\hline Entre 1 e 3 meses & $12(19,0 \%)$ & $14(22,2 \%)$ & $07(11,1 \%)$ \\
\hline De 3 a 6 meses & $06(9,5 \%)$ & $09(14,3 \%)$ & $04(6,3 \%)$ \\
\hline De 6 a 9 meses & $01(1,6 \%)$ & $11(17,5 \%)$ & $09(14,3 \%)$ \\
\hline De 9 meses a 1 ano & $01(1,6 \%)$ & $08(12,7 \%)$ & $07(11,1 \%)$ \\
\hline De 1 a 2 anos & $00(-)$ & $07(11,1 \%)$ & $04(6,3 \%)$ \\
\hline$>2$ anos & $00(-)$ & 05 (7,9\%) & 05 (7,9\%) \\
\hline
\end{tabular}

Fonte: os autores

O tempo de encaminhamento até a data da primeira avaliação foi calculado parcialmente devido à incompletude em 25 casos (39,7\%; $1-318 ; x=57,4$ dias; DP: 65,9), assim como a data do encaminhamento até a alta $(39,7 \% ; 15\} 1677$; $\mathrm{x}=339,8$ dias; $\mathrm{DP}=367,2)$.

Os resultados demonstrados na Tabela 4 são relativos, visto que a maioria dos encaminhamentos médicos entregues ao centro de saúde se encontravam sem data e outras vezes, no prontuário, havia mais de um encaminhamento sendo eles datados e outros não, sem a precisão de qual data é a inicial, não sendo considerada para cálculos nesse estudo.

O encaminhamento precoce é defendido por muitos autores como crucial pela importância no desenvolvimento do recém-nascido, principalmente nos quatro primeiros meses de vida, pois, dessa forma, a intervenção ocorrerá antes da criança desenvolver padrões incorretos de postura e movimentos alterados, obtendo assim melhores resultados. Uma vez que os bebês são encaminhados de forma tardia para os centros de saúde, já exibindo alguma incapacidade, é difícil alcançar os objetivos que seriam possíveis com o encaminhamento precoce (VIEIRA, 2016).

Um estudo realizado com 39 RN's nascidos prematuros obteve como resultado que somente cinco desses $(12,8 \%)$ foram encaminhadas para a reabilitação, referindo a carência no encaminhamento precoce para intervenção fisioterapêutica depois da alta da maternidade (CASTRO; FIGUEIREDO; CANO, 2016)
Em relação à data da avaliação fisioterapêutica até a data de alta, que define o tempo de tratamento, notase o quão variável é este por depender de vários fatores, tais como mudança de cidade dos pais, desistência do tratamento, objetivo alcançado, dificuldade de deslocamento, entre outros. Os dados relativos ao motivo da alta são apresentados no Gráfico 01.

Gráfico 1 - Dados relativos ao motivo de alta de RNs prematuros. 2015 a 2018 em um ambulatório no município em Minas Gerais (MG).

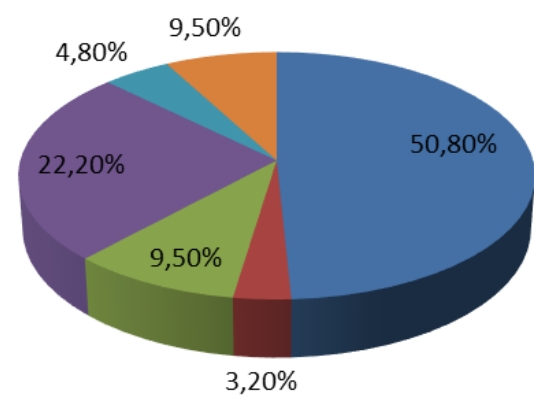

- Faltas não justificadas e/ou abandono e/ou desistência

- Mudança de cidade

não retornou após as férias

- Melhora do quadro / alcançou o objetivo

Fonte: os autores

Evidenciou-se que $50,8 \%$ das altas ocorrem por desistência ao tratamento, seja pelo número de faltas ou abandono do tratamento, contrastando com $22,2 \%$ das altas por melhora do quadro. Um estudo buscou identificar o motivo das faltas das mães dos 
RN's em atendimento fisioterápico, revelando que essas ocorrem, por várias vezes, pela falta de motivação da mãe no tratamento do filho que geralmente não pode estar presente no momento da intervenção gerando um distanciamento com a intervenção e consequente desinteresse materno (AMBROZIO, 2009).

A incompletude dos dados, principalmente aos resultados da triagem neonatal (teste do pezinho, da orelhinha e do olhinho) e a maioria dos encaminhamentos foram considerados como principais fatores limitantes desse estudo.

\section{CONCLUSÃO}

Evidenciou-se nesse estudo uma prevalência do sexo masculino de recém-nascidos prematuros nascidos de cesárea, com baixo peso ao nascer, apresentando Apgar no primeiro e quinto minuto de vida satisfatórios. As causas de prematuridade mais evidentes foram ruptura de bolsa, a préeclâmpsia/eclâmpsia/síndrome de Helps.

Faz-se necessário trabalhos pontuais de educação continuada e orientações quanto a importância do preenchimento dos prontuários, além de fazer-se relevante, também, a orientação às famílias quanto a adesão e acompanhamento ao tratamento de reabilitação promovendo a qualidade de vida dos recém-nascidos e das famílias.

\section{REFERÊNCIAS}

ABRAHÃO, Cesar Augusto França et al. Perfil do recém-nascido no serviço terapêutico de acompanhamento precoce. Journal of Amazon Health Science, v. 1, p.64-84, 2015.

AMBROZIO, Carolina Ribeiro. Participação de mães na avaliação do desenvolvimento do bebê com síndrome de down, realizada pelo psicólogo, na estimulação precoce. 2009. 156 f. Dissertação (Mestrado) - Curso de Educação, Universidade Federal do Paraná, Curitiba, 2009.

ABUD, Simone Mourão; GAÍVA, Maria Aparecida Munhoz. Análise do preenchimento dos dados sobre gravidez, parto, puerpério e recém-nascido na caderneta de saúde da criança. Revista da Sociedade Brasileira de Enfermagem e Pediatria, v. 16, ed. 1, p. 11-20, Junho 2016.
CASTRO, Gisélia Gonçalves de; FIGUEIREDO, Glória Lúcia Alves; CANO, Maria Aparecida Tedeschi.

Crianças prematuras: caracterização e intervenção fisioterapêutica. Cinergis, Franca, v. 17, p.196-201, 30 set. 2016.

CUSTÓDIO, Zaira Aparecida de Oliveira; CREPALDI, Maria Aparecida; CRUZ, Roberto Moraes.

Desenvolvimento de Crianças Nascidas Pré-Termo Avaliado pelo Teste de Denver-II: Revisão da Produção Científica Brasileira. Psicologia: Reflexão e Crítica, Florianópolis, v. 25, p.400-406, 15 mar. 2011.

FERNANDES, Pollyanna Tavares Silva et al.

Desenvolvimento neuropsicomotor de recémnascidos prematuros: uma revisão sistemática.

Conscientiae Saúde, Uberaba, v. 16, p.463-470, 21 dez. 2007.

GAíVA, Maria Aparecida Munhoz; SILVA, Fabiane Blanco da. Caderneta de saúde da criança: revisão integrativa. Revista de Enfermagem UFPE on line, Recife, v. 8, ed. 3, p. 742-749, Mar 2014.

HOLANDA, Cristyanne Samara Miranda de; ALCHIERI, João Carlos; MORAIS, Fátima Raquel Rosado; MARANHÃO, Técia Maria de Oliveira. Estratégias de desenvolvimento, acompanhamento e avaliação do atendimento da gestante no ciclo gravídicopuerperal. Revista Panamericana de Salud Pública, v. 37, ed. (6), p. 388-94., 2015.

MACHADO, Kátia Castelo Branco; TEIXEIRA, Luciana Lavor; SÁ, Fabiane Elpídio de. Perfil clínico dos recém-nascidos com retinopatia da prematuridade em um hospital público do ceará. Revista Brasileira de Pesquisa em Saúde, Fortaleza, v. 21, p.47-54, 2008.

MARCHI, Ana Clécia Maria dos Santos; GOLDBECK, Ana Stela; NUNES, Jakeline; ZAMARO, Paula Juliana Antoniazzo; CARVALHO, Tania Marini de. Triagem neonatal biológica: manual técnico. Ministério da Saúde, Secretaria de Atenção a Saúde, Brasília, v. 1, n. 80p, 2016.

MENETRIER, Jacqueline Vergutz; ALMEIDA, Gleidaiane de. Perfil epidemiológico de gestantes de alto risco com parto prematuro em um hospital de referência. Revista Saúde e Pesquisa, Maringá, v. 9, p.433-441, dez. 2016.

MENEZES, Francislene Lopes; GRACIOLI, Michelle da Silva Araújo; FREITAS, Hilda Maria Barbosa de; DIAZ, Claudia Maria Gabert; ROCHA, Bruna Dedavid da; 
GOMES, Iris Elisabete Messa; BORDIGNON, Juliana Silveira. Conhecimento das mães acerca do teste do pezinho. Espaço para a Saúde - Revista de Saúde Pública do Paraná, Londrina, v. 17, ed. 2, p. 220-228, Dezembro 2016.

MEDEIROS, Juliana Karina Brugnolli; ZANIN, Rafaela Olivetti; ALVES, Kátia da Silva (Ed.). Perfil do desenvolvimento motor do prematuro atendido pela Fisioterapia. Sociedade Brasileira de Clínica Médica, Londrina, PR, v. 7, p.367-372, 23 nov. 2009.

NASCIMENTO, Luiz Fernando Costa; GOTLIEB, Sabina Léa Davidson. Fatores de risco para o baixo peso ao nascer, com base em informações da Declaração de Nascido Vivo em Guaratinguetá, SP, no ano de 1998. Informe Epidemiológico do Sus, Brasília, v.10, ed.3, Set 2001.

NUNES, Rodrigo Dias; BERTUOL, Elisa; SIQUEIRA, Isabela Ribeiro. Avaliação dos fatores associados aos resultados neonatais no descolamento prematuro de placenta. Arquivos Catarinenses de Medicina, Palhoça, v. 4, p.11-27, dez. 2016.

OLIVEIRA, Flávia Evelyn Santos de; VIEIRA, Nayanne Martins. Causas do nascimento de recém-nascidos prematuros em uma maternidade de alto risco do município de Aracaju entre julho e dezembro de 2014. Orientador: Profa. Esp. Derijulie Siqueira de Sousa. 2015. Trabalho de conclusão de curso (Bacharel em Enfermagem) - Ciências Biológicas e da Saúde, Universidade Tiradentes, Aracaju, 2015.

OLIVEIRA, S. S. M.; MATTOS, J. G. S. Benefícios da atividade física no período gestacional. Revista Saúde e Educação, v. 1, p. 32-44, 2016.

PESSOA, Tiara Aida Oliveira; MARTINS, Christine Baccarat de Godoy; LIMA, Fernanda Cristina Aguiar; GAÍVA, Maria Aparecida Munhoz. O crescimento e desenvolvimento frente à prematuridade e baixo peso ao nascer. Avances en Enfermería, Bogota, v. 33, ed. 3, p. 401-411, 2015.

PINTO, Jessika Nauama Silva et al. Incidência de parto cesárea em uma maternidade no município de Porto Velho -RO em 2017. Revista Eletrônica Acervo Saúde, Porto Velho, v. 33, p. e1241, ago. 2019.

MINISTÉRIO DA SAÚDE. Teste da orelhinha. 2015.

MINISTÉRIO DA SAÚDE. Triagem Neonatal Biológica

Manual Técnico. 2016.
SCOPEL, Gabriela Graciolli. Avaliação do desenvolvimento motor de recém-nascidos prematuros. 2017. 80 f. Dissertação (Mestrado) Curso de Fisioterapia, Universidade Federal de Ciências da Saúde de Porto Alegre, Porto Alegre, 2017.

SILVA, Guilherme Grivicich da; SIRENA, Sergio Antonio. Perfil de encaminhamentos a fisioterapia por um serviço de Atenção Primária à Saúde, 2012. Epidemiol. Serv. Saúde, Brasília, v. 24, p.123-133, mar. 2015.

VIANA, Kelly de Jesus et al. Peso ao nascer de crianças brasileiras menores de dois anos. Cadernos de Saúde Pública, Rio de Janeiro, p.349-356, 17 out. 2012.

VIEIRA, Lindeglaciene Fernandes da Silva. A importância da estimulação precoce no recémnascido prematuro na unidade de terapia intensiva neonatal: uma revisão bibliográfica. Orientador: Esp. Jéssica Castro dos Santos. 2016. Trabalho de conclusão de curso (Bacharelado em Fisioterapia) Faculdade de Educação e Meio Ambiente, Ariquemes - RO, 2016. 